\title{
Efecto de la calidad del agua sobre macroinvertebrados acuáticos y peces presentes en la quebrada Atajo, Guapi, Cauca
}

\section{Effect of water quality on aquatic macroinvertebrates and fish present in the cause Atajo, Guapi, Cauca}

\author{
Yiskar Damián Murillo Asprilla*, Yasiri Salas Tovar*
}

\section{Resumen}

Los sistemas dulceacuícolas son uno de los recursos naturales más importantes para la vida. A pesar de su importancia, estos ecosistemas han sufrido grandes impactos causados por las actividades humanas, los cuales afectan directamente la biota acuática y la calidad de las fuentes hídricas. Considerando el valor de los macroinvertebrados acuáticos y peces como bioindicadores de calidad del agua, en esta investigación se demuestra la eficacia de estos grupos para responder a los cambios en sus hábitats. En la quebrada Atajo, mediante la metodología de muestreos ecológicos rápidos, se registraron los macroinvertebrados de los géneros Chironomus y Ablabezmyia, de la familia Chironomidae perteneciente al orden Díptero. Por su parte, la íctiofauna presentó un total de 29 individuos de las especies, Astyanax fasciatus (20) y Reniloricaria jubata, (9) perteneciente a la familia Characidae y Loricaridea respectivamente. Los datos obtenidos en esta investigación muestran que existe una relación directa entre la calidad del agua y las comunidades de macroinvertebrados y peces, porque, parámetros ecológicos como riqueza, composición y abundancia, que son característicos de fuentes con buena calidad del agua, están afectados profundamente en la quebrada el Atajo. Sin duda, es evidente la sensibilidad de estos grupos a los cambios en el entorno, donde su respuesta a las modificaciones es altamente instantánea. Los peces en la quebrada han reducido considerablementesus poblaciones, reporte que guarda estrecha relación con el panorama de los macroinvertebrados acuáticos, porque estos modelan en gran parte la ocurrencia de los peces, por ser parte fundamental de la base de la estructura trófica del agua.

Palabras clave: Calidad del agua, Cauca, Indicadores biológicos, Quebrada Atajo.
* Biólogo con Énfasis en Recursos Naturales, Instituto de Investigaciones Ambientales del Pacífico (IIAP), Quibdó, Chocó, Colombia. e-mail: ydmurillo@iiiap.org.co 


\section{Bioetnia Volumen 12, 2015}

\section{Abstract}

Freshwater systems are one of the most important natural resources for life. In spite of their importance, these ecosystems have suffered great impacts caused by human activities, which directly affect the aquatic biota and the quality of the water sources. Considering the value of aquatic macroinvertebrates and fish as bioindicators of water quality, this research demonstrates the effectiveness of these groups to respond to changes in their habitats. In quebrada Atajo, using the method of rapid ecological sampling, applying, were recorded for the macroinvertebrates, the genera Chironomus and Ablabezmyia, of the family Chironomidae belonging to the order Diptero. The cytoofauna presented a total of 29 individuals of the species, Astyanax fasciatus (20) and Reniloricaria jubata, (9) belonging to the family Characidae and Loricaridea respectively. The data obtained in this research show that there is a direct relationship between water quality and macroinvertebrate and fish communities, since ecological parameters such as richness, composition and abundance, which are characteristic of sources with good water quality, are affected deep in the ravine the Shortcut. Undoubtedly, the sensitivity of these groups to the changes in the environment is evident, where their response to the modifications is highly instantaneous. The fish in the ravine have considerably reduced their populations, a report that is closely related to the panorama of aquatic macroinvertebrates; since they largely model the occurrence of fish, as it is a fundamental part of the basis of the trophic structure of water.

Keywords: Biological indicators, Cauca, Quebrada Atajo, Water quality.

\section{Introducción}

La salud de los seres humanos, la vida silvestre y los ecosistemas dependen de los suministros adecuados de agua limpia. A pesar de esta importancia, las cuencas hídricas constantemente son objeto de contaminación y alteración, producto de diferentes actividades humanas (Wang et al., 2001). En Colombia la problemática de la contaminación en fuentes hídricas está estrechamente relacionada con el aumento exorbitante de las poblaciones que se ubican en las zonas ribereñas de los ecosistemas hídricos y con el manejo inadecuado de los desechos sólidos y líquidos producto de actividades domésticas, agrícolas, mineras entre otras, que son vertidos directamenteproduciendo una reducción sustancial de la biota acuática o incluso su desaparición (Lara-Lara et al. 2008).

Esta problemática de contaminación, se extiende a cuencas del Chocó Biogeográfico caucano, panorama que se ve agravada aún más, por la intensificación de las alteraciones a la calidad de las fuentes hídricas de esta región, situación que altera la composición y funcionamiento ecológico de los organismos que viven en los sistemas acuáticos y tienen adaptaciones únicas a hábitats específicos, microhábitats, o fuentes de alimentación, mientras que otros son cosmopolitas y aptos para sobrevivir a una amplia gama de condiciones (Ramírez et al. 2008).

Las comunidades bióticas (macroinvertebrados acuáticos y peces), han sido destacadas como indicadoras de las condiciones ambientales, porque, su presencia y/o ausencia reflejan la realidad que prevalece en el ambiente donde habitan, de las condiciones físicas, químicas y bióticas, además de las diferentes presiones sobre sus ecosistemas (Roldán 1999, Barbour et al. 1999). En los últimos años, los macroinvertebrados acuáticos y los peces han ganado protagonismo como indicadores biológicos de la calidad del agua, funcionando como una importante herramienta para monitoreo de los recursos hídricos (González y García 1995, Hurtado et al. 2005, Rosenberg y Resh 1993); esto se debe a que tales organismos responden rápidamente a variaciones ambientales y son fun- 
damentales para el entendimiento de la estructura trófica y el funcionamiento de los ecosistemas acuáticos (Lampert y Sommer 2007). Por todo lo anterior, la presente investigación tuvo como finalidad realizar un análisis del efecto que tiene la calidad del agua de la quebrada Atajo, sobre las comunidades macroinvertebradas acuáticas y peces.

\section{Metodología}

Área de estudio. El municipio de Guapi se encuentra ubicado al suroccidente del departamento del Cauca, bordeando la vertiente del Pacífico colombiano, a orillas del río Guapi, sobre una altura de $5 \mathrm{msnm}$ y una temperatura promedio de $29^{\circ} \mathrm{C}$. Este municipio limita al norte con el océano Pacífico y el municipio de Timbiquí, al este con el municipio de Timbiquí y Argelia, al oeste con el océano Pacífico y al sur con el municipio de Santa Bárbara de Iscuandé, Nariño.

Como sitio de referencia para la toma de los datos se tomó la vereda Bellavista en donde se encuentra la quebrada Atajo; las capturas de los indicadores biológicos se realizaron en el tramo aguas fluídas de esta fuente entre las coordenadas $2^{\circ} 33^{\prime} 56,7^{\prime}$ " N y 7749'21,2" W, la cual se caracterizó por presentar un promedio de amplitud de $1,30 \mathrm{~m}$ y un promedio de profundidad de $50 \mathrm{~cm}$. La temperatura del agua durante el muestreo fue $28.8^{\circ} \mathrm{C}$, presentando un cambio en su hidrogeomorfología y desvío de su cauce natural de oriente a occidente. Además, se evidenció abundante material lodoso, moderadamente sedimentado, poca vegetación ripiara, aguas de color café, materia orgánica en descomposición, hojarasca, y vegetación circundante típica de ambientes disturbados (helechos, Rebiaceae, Clusiaceae).

Métodos. Los muestreos de macroinvertebados acuáticos en la quebrada Atajo fueron dirigidos hacia ambientes estratégicos como sedimento, hojarasca, vegetación ribereña y troncos entre otros, donde, con ayuda de cernidores, bandejas plásticas y pinzas, se tomaron muestras de estos bioindicadores, que fueron depositadas en recipientes plásticos y fijadas con alcohol al $70 \%$, para su posterior identificación. Luego se trasladaron al laboratorio de Limnoligía de la Universidad Tecnológica del Chocó "Diego Luis Córdoba" (UTCH), Quibdó, donde se identificaron taxonómica hasta el taxón más accesible, con la ayuda de un estereomicroscopio, utilizando claves de Merritt y Cummis (1996), Fernández y Domínguez(2001), Posada y Roldán (2003), Domínguez et al. (2006) y Domínguez y Fernández (2009).

Por su parte, los muestreos ícticos se realizaron con ayuda de una red de arrastre con ojo de maya de $1 \mathrm{~mm}$, que consistió en realizar barridos desde la zona litoral de la quebrada abarcando todo en el espejo de agua, con el fin de garantizar las capturas de los organismos ícticos presentes en los pocos microhabitats que ofrecía el lugar. Los peces capturados fueron determinados in situ, hasta el taxón más asequible, de los cuales se eligieron tres ejemplares, que fueron depositados en bolsas plásticas transparentes con una solución de formol al 10\% con sus respectivos datos de campo (nombre regional, nombre científico, fecha, lugar de captura) y trasladados al Laboratorio de Ictiología de la UTCH, en donde fueron confirmados a partir de la utilización de las claves taxonómicas de Maldonado-Ocampo et al. (2012) y revisión de la base de datos Fishbase (1999). Además, se tomaron algunas variables físico-químicas, con el objetivo de profundizar sobre la información biológica. Para evaluar el estado de la calidad de la quebrada el atajo se realizó un análisis sobre la relación existente entre las variables biológicas y fisicoquímicas del lugar.

\section{Resultados y discusión}

En la quebradaAtajo se colectaron 2 individuos de macroinvertebrados acuáticos de los géneros Chironomus y Ablabezmyia, de la familia Chironomidae, del orden Díptero. La ocurrencia de los 


\section{Bioetnia Volumen 12, 2015}

chironómidos como únicos representantes de los macroinvertebrados en la quebrada el atajo, está relacionada con la capacidad de adaptación para habitar en ambientes alterados (Özkan 2009), como la quebrada Atajo. Si se analiza la historia de vida de este grupo, se nota que los chironómidos son organismos que soportan grandes cambios en algunas de sus variables como temperatura, $\mathrm{pH}$, oxígeno disuelto; estas características pueden ser la explicación por lo cual los chironómidos se encuentren con frecuencia en fuentes hídricas con bajas productividad, nutrientes, altos niveles de temperatura, reducción de $\mathrm{pH}$ y oxígeno disuelto (Paggi 1999), tanto así que diferentes autores (Maasri et al. 2008) consideran estos organismos indicadores de aguas ligeramente contaminadas producto de actividades antrópicas.

Adamus y Brandt (1990) afirman que la riqueza de los chironómidos se reduce con el aumento de la temperatura y la diminución del oxígeno. En este sentido, si se compara las variables fisicoquímicas (oxígeno-1.2 y temperatura-29) y los bajo datos de abundancia de los macroinvertebrados de la quebrada Atajo, se observa un patrón similar a lo que plantean los autores. Este dato permite argumentar que realmente los valores de oxígeno y temperatura son determinantes en el estado de la quebrada Atajo, porque podría influir en la dinámica ecológica de las poblaciones de los macroinvertebrados, además de otros procesos biológicos. Este argumento es corroborado por Barrenechea (2008), quien afirma que parámetros como temperatura y oxígeno son importantes para el desarrollo y mantenimiento de los organismos de primer orden de la cadena ecológica (macroinvertebrado); este mismo autor aclara, que si estas variables están por debajo de los estándares para el desarrollo de la biota, estas pueden influir en el retardo y/o aceleración de la actividad biológica, abundancia y composición de estos organismos.

Al comparar los datos de macroinvertebrados de la quebrada Atajo con los 236 individuos, 9 órdenes, 21 familias y 24 géneros encontrados en ecosistema prístinos como el río Guangüi en la misma región, se nota que existe una amplia diferencia de valores de riqueza (2 géneros) y abundancia (2 individuos). Esto quizás se relaciona con las fuertes y continuas presiones antrópicas que históricamente ha existido sobre la cuenca, lo que tal vez ha provocado modificaciones aceleradas en su hidro-morfología y entorno, lo cual ha traído consigo grandes alteraciones y reducciones a su mínima expresión de elementos esenciales para que se mantenga la diversidad y la fauna de macroinvertebrados. Karr (1999), reafirma este análisis, pues concluye que en condiciones de perturbación mínima la permanencia, composición y densidad de macroinvertebrados en una corriente es alta, lo que depende de los procesos naturales a los que su hábitat está sujeto. Sin embargo, en condiciones altamente perturbadas, tales como las que se originan a causa de acciones humanas como la contaminación, darán lugar a la degradación del ecosistema, que se refleja en descenso de los valores de riqueza y abundancia, como se ve en la quebrada Atajo.

Esta situación explica para el caso de esta quebrada, que la transformación en cauce, por el desarrollo de actividades antrópicas, genera una modificación directa de los diferentes hábitats y por ende la reducción acelerada de la riqueza y la abundancia de especies de este grupo. Lo anterior se apoya en lo expresado por García (2010), quien sostiene que la comunidad de macroinvertebrados acuáticos, además de ser afectada por la contaminación, reacciona de manera directa a los procesos de alteraciones hidromorfológicas del curso, como la remoción de sustrato y erosión de márgenes, situación similar a la quebrada muestreada, pues las observaciones realizadas en el entorno señalan que la fuente presenta síntomas de contaminación minera de oro y platino, la cual en su ejercicio remueve grandes cantidades de suelo y riberas. A este respecto, Sánchez y Mosquera (2007), manifiestan que la actividad minera causa deterioro en los ecosistemas acuáticos debido al 
aumento de sólidos totales, color, turbidez, los cuales impiden el paso de la radiación solar para la producción del oxígeno disuelto en el proceso de la fotosíntesis y a su vez limita la vida de macroinvertebrados acuáticos, situación similar a la ocurrida en la quebrada Atajo, donde elementos vitales como el oxígeno (1.2), temperatura (28.8), sólidos disueltos (4.8), turbiedad (36), y pH(3.6), se encontraron con valores por debajo de los estándares establecimiento para la vida acuática (Decreto 1594 del 1984).

Por otro lado, los resultados de abundancia, riqueza y composición íctica, en la quebrada Atajo, exhibieron datos característicos de ambientes alterados, registrándose un total de 29 individuos de las especies Astyanax fasciatus (20) y Reniloricaria jubata (9), pertenecientes a la familia Characidae y Loricaridea respectivamente; estos reportes guardan una estrecha relación con el panorama de los macroinvertebrados acuáticos en la fuente hídrica estudiada, porque estos modelan en gran parte la ocurrencia de los peces, por ser parte fundamental de la base de la estructura trófica del agua. Los resultados de los organismos ícticos en la quebrada Atajo, se pueden explicar con las postulaciones de Gallo (2003), quien afirma que la degradación del hábitat impacta negativamente a las comunidades de macroinvertebrados, lo que a su turno da lugar al decrecimiento del ciclo de nutrientes y de la producción de peces, que tienen a los macroinvertebrados como eslabón de su cadena trófica

Al comparar los datos de composición íctica de esta quebrada con estudios realizados por IIAP (2013) en cuerpos de agua de la zona media del Atrato, se observa que parámetros determinantes de la calidad biológica del agua como la riqueza (17 especies) y la composición (11 familias y 6 órdenes), fueron más altos que los reportados por este estudio con 2 especies y 2 familias (Figura 1). Esta situación se relaciona con el deterioro ambiental que refleja la quebrada Atajo, porque un ecosistemas acuático sin perturbaciones y afectaciones directas en su estructura física y trófica, con frecuencia soporta una alta riqueza y una composición diversa de sus especies.

Vale la pena anotar que aunque el ecosistema estudiado se encuentra alterado por el ejercicio de la actividad minera, la presencia de las 2 especies reportadas $A$. fasciatus y $R$. jubata (órdenes Cha-

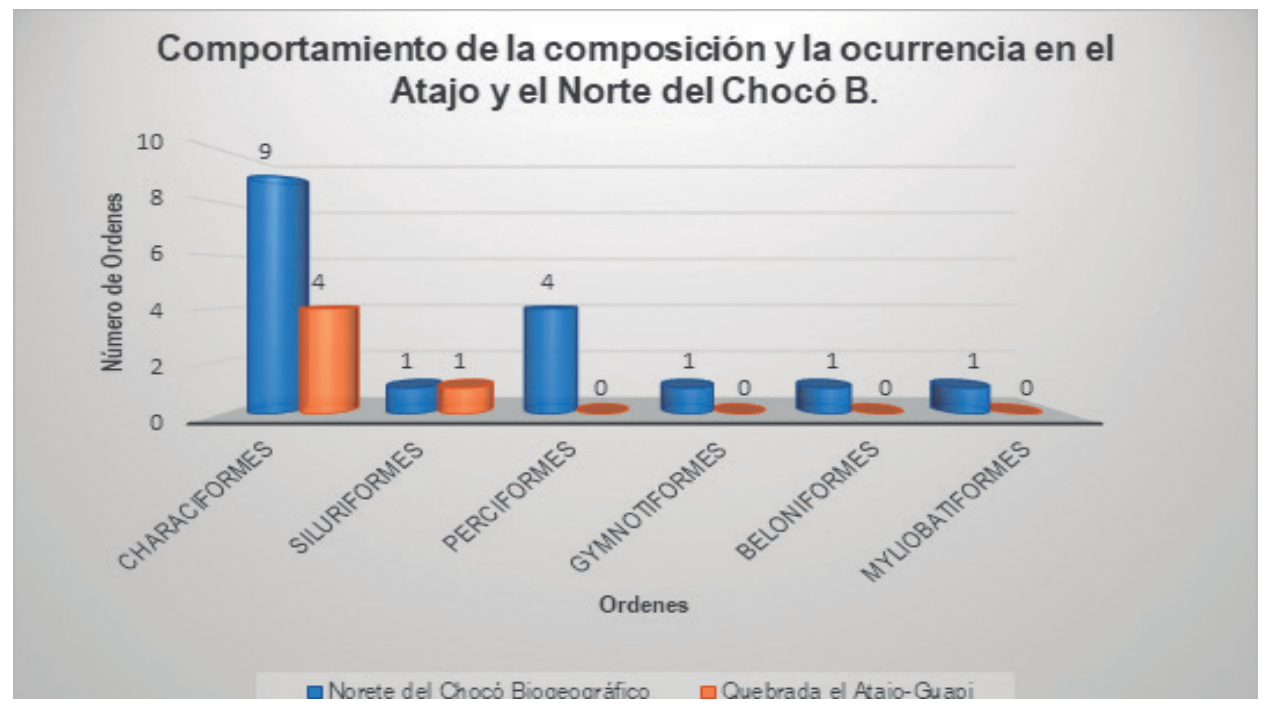

Figura 1. Comparación de presencia y ocurrencia potencial de los órdenes más representativos de los cuerpos de agua del Chocó Biogeográfico y la quebrada Atajo. 


\section{Bioetnia Volumen 12, 2015}

raciformes y Siluriformes), que generalmente son típicas de ambientes poco perturbados (Casas et al. 2005, Torres 2006, Murillo-Lozano 2011), explican que posiblemente existe una conexión con cuerpos de agua en mejor estado, lo que sumado a la amplia movilidad y al amplio espectro trófico de las especies, a estas se le facilita explorar diferente dimensiones de su nicho ecológico; además, este comportamiento puede explicar parámetros ecológicos como la abundancia de la especie Astyanax fasciatus con 20 individuos de los 29 registrados para este estudio, lo que muestran un patrón general del comportamiento gregario de la especie, guardando una estrecha relación con los reportes de abundancia de este organismo en diferentes fuentes loticas y lenticas del norte del Chocó Biogeográfico (Mojica 1999, IIAP (2013) y Lasso, 2011). Asimismo estos registros permiten entender que existe un disturbio intermedio, que auncuando se presenten alteraciones del ambiente, la intensidad del disturbio no ha sido suficiente para romper la conectividad ecológica de las especies; esto a su vez limita la entrada de especies invasoras o exóticas que modifiquen de forma total la estructura del ecosistema estudiado.

Análisis general del estado actual de la quebrada y su relación con los macroinvertebrados acuático y las comunidades de peces. Están afectados profundamente en la quebrada Atajo los parámetros ecológicos como riqueza, composición y abundancia de grupos indicadores como macroinvertebrados y peces, que guardan una estrecha relación con la buena calidad del agua; sin embargo, aunque el cauce se encuentra con algunos niveles intermedios de disturbio, ciertos sectores de la misma todavía soporta algunas especies que aprovechan los efectos de la resiliencia en recesos de la faena minera y la conectividad evidente con cuerpos de agua mayores. Es importante resaltar que existe una evidente alteración de los parámetros ecológicos de estos grupos y que al relacionarlos con las variables fisicoquímicas que también presentan un comportamiento atípico, se puede concluir que las funciones básicas del ecosistema han sido alteradas en un alto porcentaje, disminuyendo de forma evidente la salud del mismo. Es así como han sido modificados los elementos básicos autóctonos (sustratos base hojarasca, ramas, piedras y troncos, velocidad de la corriente, fluctuaciones entre aguas someras y profundas, fluctuaciones de temperatura y la disponibilidad de oxígeno) que modelan y condicionan la estructura típica de este tipo de ecosistema y por ende la supervivencia de las especies y sus atributos de riqueza, composición y abundancias, llevando a una disminución drástica de muchos de los organismos allí presentes, a la migración local de los que pueden buscar otros refugios y a la extinción de los menos aptos para sobrevivir a cambios en períodos muy cortos y en áreas muy reducidas donde el disturbio se maximiza dependiendo de la particularidad del grupo.

Basados en los anteriores análisis y partiendo de los bajos valores obtenidos en la comunidad de macroinvertebrados de la quebrada Atajo, se puede afirmar que este grupo biológico fue de suma importancia en el estudio del sistema acuático, debido a que contribuyó en el fortalecimiento de los datos fisicoquímicos, ayudando a determinar con precisión la calidad del agua, por ser muy sensibles a los cambios en el ambiente y más vulnerables a las perturbaciones antropogénicas por vivir en hábitats de pequeña escala, lo que maximiza el efecto del disturbio; esta afirmación concuerda con las aproximaciones de Swedish Environmental Protection Agency (2002), quienes manifiestan que al tener en cuenta toda una comunidad biótica se minimizan los errores y se aumenta la capacidad de detección de alteraciones en los ecosistemas acuáticos, como eutrofización, contaminación por agentes orgánicos y acidificación.

Por otro lado, no se puede dejar de lado, que la ausencia en la quebrada Atajo de elementos importantes y típicos en la composición de peces de los cuerpos de agua, tales como los Perciformes, Cyprinodontiformes loricariidae 
y Gymnotiformes, estos últimos con un amplio gradiente ecológico que le permiten responder positivamente a las alteraciones progresivas del ambiente; puede estar relacionada con una transformación acelerada del entorno, donde las modificaciones en tiempo y en espacio han sido tan rápidas que la fauna íctica autóctona es posible que ha migrado localmente por las condiciones adversas y se han establecido en cuerpos de agua adyacentes a la quebrada Atajo.

\section{Conclusión}

Las comunidades bióticas registradas en esta investigación, muestran la alta sensibilidad para responder a las modificaciones en su ambiente, donde parámetros ecológicos como riqueza, composición y abundancia que guardan una estrecha relación con la calidad buena del agua, están afectados profundamente en la quebrada Atajo. La poca presencia de especies intolerantes a la contaminación, indican que algunas de las variables fisicoquímicas se encuentran fuera de sus límites de tolerancia y afectan sus ciclos de vida, por lo que seguramente migraron a ecosistemas con mejores condiciones. En este sentido se puede concluir que la quebrada Atajo no presenta condiciones óptimas para mantener altas poblaciones de organismos acuáticos, por lo tanto el sistema estudiado se puede considerar como un ambiente con alteraciones de sus propiedades físicas y químicas.

\section{Literatura citada}

Adamus PR, Brandt K. 1990. Impacts on quality of inland wetlands of the United States: A survey of indicators, techniques, and applications of community level biomonitoring data. EPA/600/3-90/073 (NTIS PB 113 837/AS). Corvallis: USEPA Environmental Research Lab; 406 pp.

Barbour MT, Gerritsen J, Snyder BD, Stribling JB. 1999. Rapid bioassessment protocols for use in streams and wadeable rivers: periphyton, benthic macroinvertebrates and fish. Second ed. Washington, DC: EPA
(United States Environmental ProtectionAgency). URL disponible en: https://archive.epa.gov/water/archive/ web/html/ch09main.html

Barrenechea A. 2008. Aspectos fisicoquímicos de la calidad del agua. Capítulo I. URL disponible en: http:// www.bvsde.ops-oms.org/bvsatr/fulltext/tratamiento/ manualI/tomoI/uno.pdf

Casas Y, Carrascal O, Rivas TS, Lozano Y. 2005. Composición y diversidad íctica en la quebrada Chaparraidó, sistema hídrico del Medio Atrato, Chocó, Colombia. Rev Institucional UTCH. 22 (2): 58-64.

Domínguez E, Molineri C, Pescador ML, Hubbard MD, Nieto C. 2006. Ephemeroptera of South America. Aquatic Biodiversity in Latin America. v.2. Sofia: Pensoft Pub; 646 pp.

Domínguez E, Fernández HR. 2009. Macroinvertebrados bentónicos sudamericanos. Sistemática y biología. Tucumán: Fundación Miguel Lillo; 654 pp. URL disponible en: https://www.researchgate.net/publication/260417584_Macroinvertebrados bentonicos_Sudamericanos_Sistematica_y_Biologia

FishBase. 1999. FishBase 99 CD-ROM. ICLARM, Manila.

Fernández HR, Domínguez E. 2001. Guía para la determinación de los artrópodos sudamericanos. Tucumán: Universidad Nacional de Tucumán, Facultad de Ciencias Naturales e Instituto M. Lillo.

Gallo EL. 2003. The importance of stream invertebrates to riverine ecosystem function. Ecology \& Geomorphology of Streams: The Scott River Study. UC Davis, Department of Geology. URL disponible en: https://webcache.googleusercontent.com/search?q=cache:S1ccgZnP1XoJ:https://watershed.ucdavis.edu/ education/classes/files/content/flogs/Erika_Gallo. $\mathrm{pdf}+\& \mathrm{~cd}=2 \& \mathrm{hl}=\mathrm{es} \& \mathrm{ct}=\mathrm{clnk} \& \mathrm{gl}=\mathrm{co}$

García MT. 2010. Estudio integral del estado ecológico de la cuenca del río Lagares. Xunta de Galicia: Dirección Xeral de Consevación da Natureza. 102 pp. URL disponible en: http://mediorural.xunta.gal/fileadmin/ arquivos/publicacions/pesca/Estudio_estado_ecologico cuenca del Lagares.pdf

González M, García D. 1995. Restauración de ríos y riberas. Madrid: Fundación Conde del Valle de Salazar, Escuela Técnica Superior de Ingenieros de Montes, Universidad Politécnica de Madrid; 319 pp.

Hurtado S, García F, Gutiérrez PJ. 2005. Importancia ecológica de los macroinvertebrados bentónicos de la subcuenca del río San Juan, Querétaro, México. Folia Entomol Mex. 44 (3): 271-86. URL disponible en: http://www.redalyc.org/articulo.oa?id=42444301

Instituto de Investigaciones Ambientales del Pacífico. 2013. Caracterización ecológica del complejo cenagoso la Honda, Tangui, Medio Atrato, Chocó. Informe final. 


\section{Bioetnia Volumen 12, 2015}

Quibdó: IIAP; 102 pp.

Karr JR. 1999. Defining and measuring river health. Freshwater Biol. 41 (2): 221-34. URL disponible en: http://onlinelibrary.wiley.com/doi/10.1046/j.13652427.1999.00427.x/abstract

Lampert W, Sommer U. 2007. Limnoecology: The ecology of lakes and streams. 2a ed. New York: Oxford University; $324 \mathrm{pp}$.

Lara-Lara JR, Arreola JA, Calderón LE, Camacho VF, Espino Gde la L, Escofet A, et al. 2008. Los ecosistemas costeros, insulares y epicontinentales. En: Capital natural de México. Vol. I: Conocimiento actual de la biodiversidad. Ciudad de México: Comisión Nacional para el Conocimiento y Uso de la Biodiversidad (Conabio); pp. 109-34. URL disponible en: http:/www. biodiversidad.gob.mx/pais/pdf/CapNatMex/Vol\%20I/ I04 Losecosistemascos.pdf

Maasri A, Fayolle S, Gandouin E, Garnier R, Franquet E. 2008. Epilithic chironomid larvae and water enrichment: is larval distribution explained by epilithon quantity or quality? J North Am Benthol Soc. 27 (1): 38-51. URL disponible en: http://www.bioone.org/doi/ pdf/10.1899/07-013R1.1

Maldonado-Ocampo JA, Ortega-LaraA, Usma JS, Galvis G, Villa-Navarro FA, Vásquez L, et al. 2005. Peces de los Andes de Colombia. Guía de campo. Bogotá: Instituto de Investigación de Recursos Biológicos “Alexander von Humboldt"; 346 pp. URL disponible en: $\underline{\text { http:// }}$ awsassets.panda.org/downloads/peces de los andes_de_colombia.pdf

Merritt RW, Cummins KW (eds.). 1996. An introduction to the aquatic insects of North America. $3^{\text {a }}$ ed. Berkeley: University of California; 862 pp. URL disponible en: http://coweeta.uga.edu/publications/221.pdf

Ministerio de Agricultura. 1984. Decreto 1594 del 1984. Bogotá: MinAgricultura; 55 pp. URL disponible en: http://www.alcaldiabogota.gov.co/sisjur/normas/Norma1.jsp? $\mathrm{i}=18617$

Mojica JI 1999. Lista preliminar de las especies de peces dulceacuícolas de Colombia. Rev Acad Colomb Cienc Exac Fis Nat. 23: 547-66.

Murillo Y, Lozano O. 2011. Modelo ícticos piloto de monitoreo de calidad de agua en una microcuenca del alto San Juan, Chocó, Colombia. Tesis de pregrado. Quibdó: Universidad Tecnológica del Chocó.

Özkan N, Moubayed-Breil J, Camur-Elipek B. 2010.
Ecological analysis of chironomid larvae (Diptera, Chironomidae) in Ergene River basin (Turkish Thrace). Turk J Fish Aquat Sci. 10: 93-9. URL disponible en: http://www.trjfas.org/uploads/pdf 383.pdf

Paggi AC. 1999. Los Chironomidae como indicadores de calidad de ambientes dulceacuícolas. Rev Soc Entomol Argent 58 (1-2): 202-7. URL disponible en: https://webcache.googleusercontent.com/search?q=cache:7vq-rgDiwW8J:https://www.biotaxa.org/RSEA/ article/download $/ 32770 / 29095+\& \mathrm{~cd}=2 \& \mathrm{hl}=\mathrm{es} \& \mathrm{ct}=-$ $\mathrm{clnk} \& \mathrm{gl}=\mathrm{co}$

Posada JA, Roldán G. 2003. Clave ilustrada y diversidad de las larvas de Trichoptera en el nor-occidente de Colombia. Caldasia. 25 (1): 169-92. URL disponible en: http://www.bdigital.unal.edu.co/37582/1/39407175363-1-PB.pdf

Ramírez DA, Valladares F, Blasco A, Bellot J. 2008. Effects of tussock size and soil water content on whole plant gas exchange in Stipa tenacissima L.: Extrapolating from the leaf versus modelling crown architecture. Environ Experime Bot. 62 (3): 376-88. URL disponible en: http://www.sciencedirect.com/science/article/pii/ $\underline{\text { S0098847207001839 }}$

Roldán G. 1999. Los macroinvertebrados y su valor como indicadores de la calidad del agua. Rev Acad Colomb Cienc 23 (88): 375-87. URL disponible en: https:// es.scribd.com/document/66696971/Los-Macroinvertebrados-y-Su-Valor-Como-Indicadores-de-La-Calidad-Del-Agua

Sánchez CY, Mosquera MD. 2007. Evaluación del efecto generado por la actividad minera sobre la comunidad de macroinvertebrados acuático en la quebrada San Antonio Tadó, Chocó. (Tesis de grado). Quibdó: Universidad Tecnológica del Chocó.

Torres Y, 2006. Estudio preliminar de algunos aspectos ambientales y ecológicos de las comunidades de peces y macroinvertebrados acuáticos en el río Tutunendo, Chocó, Colombia. Rev Acad Colomb Cienc Exac Fis Nat. 30 (114): 67-76. URL disponible en: http:// catalogo.unisucre.edu.co/cgi-bin/koha/opac-detail. pl?biblionumber $=18247$

Wang L, Lyons J, Kanehl P, Bannerman R. 2001. Impacts of urbanization on stream habitat andfish across multiple spatial scales. Environ Manage. 28 (2): 255-66. URL disponible en: https://www.ncbi.nlm.nih.gov/ pubmed/11443388 